\title{
Systems medicine: a real approach for future personalized oncology?
}

\section{"...[the] bigger challenge will be to explore and understand how the cancer genome functions as a complex whole-biological system..."}

Current and emerging biomedical science efforts are driven by determining how to improve clinical outcomes for patients, but the roadmap to achieve this goal has been controversial. More than standard medicine, systems medicine attracts increasing research interest for some sophisticated diseases such as cancer because it might lead to improved clinical results more quickly and efficiently. High-throughput technology has revolutionized the area of translational research, confirming the high complexity and heterogeneity of common diseases, particularly cancer. Therefore, moving from 'classic' single-gene-based molecular investigation to molecular network research might result in discovering clinical implications faster and more efficiently [1-3].

The greatest challenge yet, however, is how to integrate molecular engineering and computing to predict complex systems inference, such as tumorigenesis and metastasis. As the cost of reading and writing DNA in the last decade has dropped 1 million-fold and high-quality oncological outcomes data (recurrence-death vs survival) has become available, efforts are focused on how, when using reverse engineering from clinical data, to discover the underlying key DNA changes, and to understand the functions and interactions responsible for phenotypic heterogeneity (treatment response/cure versus drug resistance/death) [4]. Here we explore the perspectives of the systems approach for the development of molecular network based cancer targets [4], and challenges to their translation into future day-to-day clinical practice.

The emerging evidence is conclusive and leaves no room for debate. Cancer initiation, growth and metastasis are far more sophisticated biological processes than we thought. Indeed, extensive genetic studies $[5,6]$ and next-generation DNA sequencing technology-based whole-cancergenome sequencing studies [7] on lung and breast cancer, and for the first time, melanoma [8-11], confirm the high complexity and heterogeneity of cancer. These findings allow modest optimism regarding the efficiency of the present generation of biologic drugs, despite some controversy over responsiveness in advanced disease. For the vast majority of biologics, the goal of targeting just one single component (mutated gene) or an entire signaling pathway, has had modest practical success. Indeed, the results of clinical trials in the metastatic setting have demonstrated either no survival prolongation or only a progression-free survival (PFS) improvement with no significant benefit in overall survival (OS) [4]. Successful translation of biologics from the metastatic setting to the adjuvant setting has been demonstrated only for trastuzumab in patients with hEGF receptor type 2 (HER2) early-stage breast cancer. However, even here longer followup results are required to assess whether targeted agents simply have a recurrence-delaying effect or if they can result in a true cure.

“...moving from 'classic' single gene based molecular investigation to molecular network research might result in discovering clinical implications faster and more efficiently.'

Adjuvant systemic treatment in cancer improves survival. Over the last few years, adjuvant chemotherapy after complete surgical tumor resection (R0) has saved the lives of many patients with stage II and III solid cancer.

However, recurrence and death rates are still alarmingly high [12]. Treatment failure rates range between $35 \%$ for colorectal cancer, for example [13], and $80 \%$ or even higher for aggressive cancers such as pancreatic or lung cancer [12]. Although modern adjuvant chemotherapybased response rates are approximately $30 \%$, the absolute net gain of adding chemotherapy, compared with surgery alone, affects long-term OS by approximately $10 \%$, ranging between 2 and $15 \%$ depending on cancer type, tumor stage

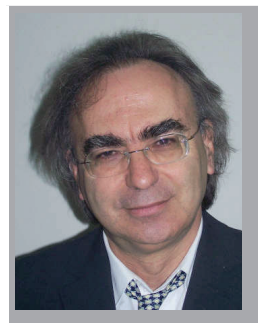

Dimitrios H Roukos

Personalized Cancer Medicine

Biobank, Department of

Surgery, loannina University

School of Medicine, Ioannina,

45110 Greece

Tel.: + +302651007423

Fax: +30 2651007094

droukos@uoi.gr 
and other clinicopathologic and therapeutic variables. How could these substantial failures rates be improved?

The advent of targeted therapy over the last decade has created overenthusiasm. It is only recently that the concept of biologic agents has been incorporated into the pharmaceutical industry, approximately 50 years after the discovery of the EGF and its receptor (EGFR). Targeting only cancer cells but not healthy cells when using biologics could dramatically improve the poor outcomes of cancer patients, while maintaining very low adverse effect profiles. Now, as multiple sets of data from randomized controlled trials have been made available, how optimistic have we been?

\section{"Integrating all these data together with cancer genetics and functional data ... into molecular networks, could potentially approach a genotype-phenotype map.'}

As the list of biologic agents approved by the US FDA for many common solid cancers grows rapidly, so too do questions and uncertainty for day-to-day practice. Clinical success is limited in both the metastatic and adjuvant settings. In stage IV disease, most of the biologic agents that have been approved by the US FDA have been based primarily on significant PFS improvement. However, the objectivity of PFS without any significant OS benefit for assessing the efficacy of inhibitors of signaling pathways, such as EGFR (cetuximab or panitumab) or angiogenesis (bevacizumab), has been questioned. For example, even for selected patients with $K R A S$ wild-type metastatic CRC, the addition of cetuximab to irinotecan, fluorouracil and leucovorin (FOLFIRI) significantly increased RFS only and not OS [14]. Similar serious concerns have emerged for VEGF inhibitors, which suggests bevacizumab limitations for various solid cancers [15]. In the adjuvant setting, potential life-saving effects have been reported only for the anti-HER2 antibody trastuzumab for HER2-positive early-stage breast cancer. Even for this isolated success, the net response rate of the addition of this agent to chemotherapy is only $10 \%$. Longer follow-up results should be awaited before drawing robust conclusions about whether trastuzumab can have long-term effects resulting in cure.

In the prevention setting, personalized preventive intervention at the right time in high-risk persons within the general population remains elusive. Genetic testing allows the identification of heritable breast cancer or colorectal cancer, but these inherited syndromes account for only $3-10 \%$ of these cancer types, the predictive accuracy at age 50 years is less than $50 \%$, and ultimately, $25 \%$ of carriers of mutation in $B R C A 1 / 2$ or mismatch-repair genes will never develop breast cancer or colorectal cancer respectively. This uncertainty is a challenge to a tailored surgical or medical preventative intervention in these high-penetrance mutations carriers. In the post-genomic era, genomewide association studies (GWAS) confirm the results of traditional single-gene research. First, common genetic variation seems to have only a limited role in determining people's predisposition to many common diseases. Second, gene variants that are very rare in the general population can have large effects on predisposition. For example, rare mutations that cause the elimination of chunks of the genome can raise the risk of diseases such as schizophrenia, epilepsy or autism by up to 20-fold [16]. Currently, for the vast majority of cancer cases, neither the risk of cancer development nor the risk of treatment failure can be predicted in an individual person or patient, respectively.

How can we explain these disappointing results provided by both single-gene-based studies and personal genomics via GWAS using high-throughput screening?

First, by the highly complex nature of cancer genetics and genomics. All cancers are driven by mutations. Over the last quarter of a century, several sequencing strategies have identified multiple mutations in approximately 400 cancer genes [101]. Given the large number of mutations accumulated in the long human evolutionary history, the exact number of genetic alterations and cancer genes must be substantially larger and difficult to estimate [7]. The catalogue of genetic alterations and cancer genes implicated in each cancer type is expected to be completed by using next-generation DNA-sequencing technology over the next decades, at least for the most common cancer types responsible for current high-mortality rates. However, many challenges remain to be overcome. The costs for complete cancer-genome sequencing remain high. To obtain a complete catalogue of mutations from an individual human cancer may require 20 -fold sequence coverage of the cancer genome, increasing the costs. More than 100,000,000,000 bp of DNA sequence will probably be required to identify the catalogue of mutations in a single cancer genome [7]. Beyond point mutations, including base substitutions and small insertions 
or deletions, genomic rearrangements in which DNA has been broken and then rejoined to a DNA segment from elsewhere in the genome, and copy-number changes may play an important role in predicting cancer and thus should be identified [11]. Ultimately, when compiling the complete catalogue of mutations, these should be distinguished as being somatic versus inherited as well as into causal (driver) versus noncausal (passenger) [7].

The second and bigger challenge will be to explore and understand how the cancer genome functions as a complex whole-biological system in individual patients, in relationship to environment, lifestyle and genetic heritability. Next-generation DNA-sequencing technologies, including whole or partial targeted genome sequencing, genome-wide RNA, serial analysis of gene expression (SAGE), microRNAs, protein-DNA interactions, and comprehensive analyses of transcriptomes and interactomes $[1,2,6,7]$ will provide important insights into individual cancer cell signaling pathway networks and interactions among cancer cells and their microenvironment. An improved understanding of this intracellular downstream pathway network [17] is essential for discovering cancer targets, and for developing biomarkers and biologic agents [4].

Third, the varied nature of tumor heterogeneity and activated signaling pathways plays a role [6]. Indeed, because of the large repertoire of mutations, causal genetic alterations and cancer genes vary widely among patients with the same clinicopathologic features and tumor node metastasis staging. For example, beyond the EGFR and VEGF signaling pathways, others, including Wnt $-\beta$ catenin, Notch, p53, TGF, Hedgehog and several other pathways, may have a crucial role in signal transduction from cells outside to the nucleus, resulting in tumorigenesis and metastasis $[2,18]$. A recent study using modern high-throughput screening technology revealed that a median of approximately 12 signaling pathways are deregulated in pancreatic cancer [6]. This finding makes the absence of efficacy of the present generation of biologics unsurprising since they usually target a single pathway, such as the EGFR family (EGFR and HER2), VEGF, or combinations of both. Potentially, such a treatment could be effective if there were novel biomarkers to predict a patient's tumor with the activation of only these downstream pathways. Overall, however, this state is much more complicated if we consider that, within individual solid tumors, cancer cell subpopulations often vary with regard to their sensitivity or resistance to systemic treatment with chemotherapy and biologic agents or radiotherapy [19]. Thus, for most patients, particularly those with advanced solid tumors, systems biology approaches are required.

Given this wide heterogeneity and the high complexity of cancer [2,4-6], two major strategic areas are now taking shape. First, one realistic approach is reflected by the current standards, which suggest awaiting the availability of hundreds or thousands of whole-cancer genome sequence. Second, prospective studies will be required to compare DNA variants and functional data. This comparative effectiveness research is essential to identify causal mutations linked with cancer initiation and/or metastasis. Although this strategy is rational for the development of robust markers and biologics, several decades will be needed. Alternatively, sophisticated approaches are proposed to shorten this time.

\section{"Molecular systems approaches allow progress towards understanding how intracellular signaling pathways networks operate and how interactions among heterogeneous cancer cells within an individual primary tumor and its associated metastases govern the oncological outcomes."}

Network-based approaches represent one of the most rational and promising strategic areas. Although more exotic, the systems approach is unavoidable if we aim for faster clinical success. In the context of biological systems, a network can be viewed as a graphical model that represents relationships among DNAs, RNAs, proteins, metabolites and higher order phenotypes such as cancer. In this way, networks provide a way to visualize extremely large-scale, complex relationships among the molecular interactions underlying a specific cancer phenotype. The complexity of a large-scale set of DNA and RNA variants and the protein interactions in time and space, which is related to phenotypic traits in living systems [1], such as human cancer genomes, requires a systems approach and network models to understand this complex genotype-phenotype relationship. Modeling the extent of such relationships between molecular entities, between heterogeneous cancer cells within a primary tumor with differing response to therapeutics, and between metastatic cancer cells in secondary tumors at other organs (metastases) is a daunting task. Given the millions of complex molecular interactions 
in timely dynamic biological processes, oncological outcomes (cancer initiation and metastases) could be predicted only by integrating such largescale datasets into mathematical algorithms and powerful computational strategies [1-3].

Ideally, to construct predictive models, a complete set of both cancer causal mutations and functional data are required. Over recent years, a large amount of such data has been collected, but the catalogue of cancer genes and driver (causal) genetic alterations - point mutations, rearrangements and copy-number changes - for each solid cancer will still require a lot of time to complete, despite the reduced costs of next-generation sequencing.

\section{"Novel, network-based targets should prove their potential superiority over the current standard cancer diagnostics and therapeutics in clinical trials."}

As an alternative, the complex genotype-phenotype relationship could be predicted more quickly by using reverse engineering. For example, complete, high-quality clinicopathologic, tumor staging, treatment and follow-up data, which define the phenotypic outcome (recurrence/death vs survival/cancer cure), are available for some cancer types. Integrating all these data together with cancer genetics and functional data, which, for several cancers, such as breast cancer or colorectal cancer, are nearly complete, into molecular networks could potentially approach a genotype-phenotype map.

Although these systems approaches are still in their infancy, they open a rational way towards network-based identification of crucial biomarkers and biologics.

In summary, the results of clinical trials testing biomarkers and biologics developed on the basis of conventional single-gene cancer research have demonstrated modest isolated clinical success. These findings are not surprising given the molecular network complexity and heterogeneity of cancer. In the post-genomic era, next-generation
DNA-sequencing technology-based results confirm available evidence that cancer initiation, growth and metastasis are driven by molecular networks rather than just one mutated gene or a single deregulated signaling pathway.

\section{Future perspective}

Molecular systems approaches allow progress towards understanding how intracellular signaling pathways networks operate and how interactions among heterogeneous cancer cells within an individual primary tumor and its associated metastases govern the oncological outcomes. This comprehensive understanding of how a solid tumor functions as a whole biological system, including the primary tumor, its associated metastases and their relationships with multiple host variables, such as heritable causal mutations, environmental exposure and lifestyle, can be achieved by systems approaches, revealing the fundamental importance of systems medicine. Therefore, such sophisticated network-based approaches represent a major hope for the development of novel robust biomarkers and effective biologics.

In the real world, the principles and rules of comparative effectiveness research and the stage of FDA approval should be considered at an early preclinical development stage of designing such molecular systems-based markers and drugs, giving particular emphasis to the integration of clinical data. Novel, network-based targets should prove their potential superiority over the current standard cancer diagnostics and therapeutics in clinical trials.

\section{Financial \& competing interests disclosure}

The author has no relevant affiliations or financial involvement with any organization or entity with a financial interest in or financial conflict with the subject matter or materials discussed in the manuscript. This includes employment, consultancies, honoraria, stock ownership or options, expert testimony, grants or patents received or pending, or royalties.

No writing assistance was utilized in the production of this manuscript.

\section{Bibliography}

1 Schadt EE: Molecular networks as sensors and drivers of common human diseases. Nature 461(7261), 218-223 (2009).

2 Roukos DH: Novel clinico-genome network modeling for revolutionizing genotypephenotype-based personalized cancer care. Expert Rev. Mol. Diagn. 10(1), 33-48 (2010).

3 Deisboeck TS: Personalizing medicine: a systems biology perspective. Mol. Syst. Biol. 5, 249 (2009).
4 Roukos DH: Mea Culpa with cancer-targeted therapy: new thinking and new agents design for novel, causal networks-based, personalized biomedicine. Expert Rev. Mol. Diagn. 9(3), 217-221 (2009).

5 Wood LD, Parsons DW, Jones S: The genomic landscapes of human breast and colorectal cancers. Science 318(5853), 1108-1113 (2007).

6 Jones S, Zhang X, Parsons DW: Core signaling pathways in human pancreatic cancers revealed by global genomic analyses. Science 321(5897), 1801-1806 (2008).
7 Stratton MR, Campbell PJ, Futreal PA: The cancer genome. Nature 458(7239), 719-724 (2009).

8 Pleasance ED, Stephens PJ, O'Meara S et al.: A small-cell lung cancer genome with complex signatures of tobacco exposure. Nature 463(7278), 184-190 (2009).

9 Pleasance ED, Cheetham RK, Stephens PJ et al:: A comprehensive catalogue of somatic mutations from a human cancer genome. Nature 463(7278), 191-196 (2010). 
10 Shah SP, Morin RD, Khattra J et al.: Mutational evolution in a lobular breast tumour profiled at single nucleotide resolution. Nature 461(7265), 809-813 (2009).

11 Stephens PJ, McBride DJ, Lin ML et al.: Complex landscapes of somatic rearrangement in human breast cancer genomes. Nature 462(7276), 1005-1010 (2009).

12 Jemal A, Siegel R, Ward E, Hao Y, Xu J, Thun MJ: Cancer statistics, 2009. CA Cancer J. Clin. 59(4), 225-249 (2009).

13 Sargent D, Sobrero A, Grothey A et al: : Evidence for cure by adjuvant therapy in colon cancer: observations based on individual patient data from 20,898 patients on 18 randomized trials. J. Clin. Oncol. 27(6), 872-877 (2009).

14 van Cutsem E, Köhne CH, Hitre E et al.: Cetuximab and chemotherapy as initial treatment for metastatic colorectal cancer. N. Engl. J. Med. 360 (14), 1408-1417 (2009).

15 Ellis LM, Reardon DA: Cancer: the nuances of therapy. Nature 458(7236), 290-292 (2009).

16 Goldstein DB: 2020 visions. Personalized medicine. Nature 463, 27 (2010).

17 Hahn WC, Weinberg RA: Modelling the molecular circuitry of cancer. Nat. Rev. Cancer 2(5), 331-341 (2002).
18

Peterson RT: Drug discovery: propping up a destructive regime. Nature 461(7264), 599-600 (2009).

19 Bastiaens P: Systems biology: when it is time to die. Nature 459 (7245), 334-335 (2009).

\section{Website}

101 Cancer Gene Census www.sanger.ac.uk/genetics/CGP/Census/ 